\title{
A Study of the Extended Forecasting with the Barotropic Model
}

\author{
By S. Kubota \\ Meteorological Research Institute, Tokyo \\ and Y. Kurihara \\ Central Meteorological Observatory, Tokyo
}

(Manuscript received 7 May 1956)

\begin{abstract}
In order to obtain the materials appropriate for the study of extended forecasting, we calculated the six-day change of the height of $500-\mathrm{mb}$ isobaric surface by the barotropic model, where the height $z$ is expressed by one-dimensional Fourier coefficients along each latitude circle.
\end{abstract}

\section{§ 1. Introduction}

As soon as we wish to extend the forecasting period, by the method of physical weather prediction, many difficulties appear in our way; some are of physical nature and the others of technical nature. The effects of friction, radiation, topography etc. up on the change in vorticity belong to the former problem, and computational instability, boundary conditions etc. to the latter.

Physical questions have to be solved substantially some other time, but at present some technical questions which must appear in the computation for a large area, even with the barotropic model, should be elucidated before proceeding further.

Now, the fact is that few has succeeded even in the medium range forecasting, especially due to the difficulties of finding the appropriate model and method suitable for it.

Hubert (1951), Berson (1952) and Clapp (1952) have computed the height change of $500 \mathrm{mb}$ isobaric surface, using a simple vorticity equation and expressing the height by $z=\hat{z}+z^{\prime}$ in which $\hat{z}$ denotes preceeding five-days mean value of $z$ for each day, and $z^{\prime}$ the deviation from the former. The following two assumptions which they used in these methods seem to be not acceptable, i.e., $\hat{z}^{\prime}=0$ is assumed, and $\boldsymbol{\nabla} \cdot \widehat{\eta^{\prime} \boldsymbol{v}^{\prime}}$ and other terms like this are assumed to be dealt with statistically without any reason. Accordingly these methods cannot be expected to develop favourably in future.
Meanwhile, K. Gambo and M. Aihara (1954), and Y. Masuda (1955) have attempted the application of Fjortoft's method to the long range forecasting. The results obtained by them, however, seem to be unsatisfactory, because they are now unable to obtain the stable field proper and effective for a long range forecasting.

Y. Sasaki (1955) deviced the method of numerical prediction based on the variational principle and attempted to apply his method to a long range forecasting. This method seems to have some advantages over others, as he looked for a conservative quantity suitable for a long range forecasting and it is shown to be valid to deal with integrated vorticity, for example, as a vortex filament, just like Clapp did before.

And, the possibility of numerical prediction of $500 \mathrm{mb}$ isobaric surface with the barotropic model in a long range forecasting was investigated by Gambo (1955) and Uchiyama (1956). The former studied the problems of computational instability and truncation error which will appear in extending the forecasting period, and the latter, investigating the conservation of vorticity, has arrived at nearly the same result with the former concerning the perspective of the limitation of the period of extended forecasting by the method similar to the current ones. Considering their results it will be reasonable to compute the vorticity change in three days or more, possibly up to seven days.

Now, the space mean of a pressure pattern 
is recongnized to be superior to its time mean in the physical weather forecasting in many respects, especially in a medium range forecasting. However, inspite of laborious studies to find a good form of space mean, e.g., by S. Syōno, the conclusive result has not yet been obtained. Neglection of the waves, the wave number of which are larger than certain number $n_{0}$, is assumed in our method to correspond to the special smoothing process of an isobaric surface. The determination of $n_{0}$ is not based upon the solid physical foundation, the study of which shall be left in future.

Next, let us summarize the concept of our method in the following;

if we put

$$
z=\sum_{n=0}^{\infty} z_{n}=\sum_{n=0}^{\infty}\left\{\overline{z_{n}}+\left(z_{n}-\overline{z_{n}}\right)\right\}
$$

where $\overline{z_{n}}$ denotes the meridionally smoothed values of $z_{n}$, then through the substitution

$$
\begin{gathered}
z^{*}=\sum_{n=0}^{n_{0}} \overline{z_{n}}, \\
z^{\prime}=\sum_{n=n_{0}+1}^{\infty} \overline{z_{n}}+\sum_{n=0}^{\infty}\left(z_{n}-\overline{z_{n}}\right)
\end{gathered}
$$

the following equation is derived:

$$
\begin{aligned}
\frac{f}{g} \frac{\partial \zeta}{\partial t}= & J\left(\eta^{*}, z^{*}\right)+J\left(\eta^{*}, z^{\prime}\right) \\
& +J\left(\eta^{\prime}, z^{*}\right)+J\left(\eta^{\prime}, z^{\prime}\right)
\end{aligned}
$$

where $\eta^{*}$ and $\eta^{\prime}$ are the vertical components of absolute vorticity corresponding to $z^{*}$ and $z^{\prime}$ in the equation (1.2) respectively, and $J$ denotes Jacobian.

It is exemplified that the terms $J\left(\eta^{*}, z^{\prime}\right)$ and $J\left(\eta^{\prime}, z^{\prime}\right)$ in the eqution (1.3) are generally small compared with the order of other two terms and their variation with time is thought to be rapid. Then, the principal part of contribution for the long range variation in the atmosphere is considered to be due to $J\left(\eta^{*}\right.$, $\left.z^{*}\right)$, and the effect due to $J\left(\eta^{\prime}, z^{*}\right)$ should be taken into account, if necessary. From another point of view, if we can put $z^{\prime}$ as large as the observing or reading error of $z$, and make the distribution of $z^{\prime}$ at random and small scale over a region, the terms containing $z^{\prime}$ may be omitted.

Lastly it should be added that this method has the following characteristics, ramely, (1) the disturbances are always smoothed more than necessary, (2) some close connection is forced to exist even between two disturbances, and this is regarded to be not avoided in the method by Fourier series, but may be profitable if the above close connection should exist actually in the atmosphere, (3) bad influence from the boundaries reaches far inside the forecasting area, (4) the extension of the forecasting area in order to extend the forecasting period and obtaining the accurate expression of vorticity field of the small scale disturbances are incompatible, and (5) the change in $A_{0}(\varphi)$ (zonal mean field) cannot be computed by our method, which is to be touched upon later. This last problem, together with determination of critical wave number $n_{0}$, shall be left for the study in future.

\section{§ 2. Method of integration of Vorticity Equation}

Formerly, we deviced the method of integration of vorticity equation (S. Kubota 1954, Y. Kurihara 1955), that is, the so-called quasi-double Fouries Series Method or Method of one-dimensional Fourier Series with onedimensional Relaxation. Here this method is adopted with some revisions. We shall present here the main line of this method.

Expanding the height $z$ of $500 \mathrm{mb}$ isobaric surface into one-dimensional Fourier series

$$
z=\sum_{n=1}^{\infty}\left(A_{n} \cos n \lambda+B_{n} \sin n \lambda\right)
$$

along each latitude circle, and putting into the vorticity equation

$$
\frac{\partial \zeta}{\partial t}=-v \cdot \nabla \eta
$$

$$
\text { *) } \begin{aligned}
u & =-\frac{g}{f} \frac{\partial z}{a \partial \varphi} \\
& =-\frac{g}{f a}\left\{\sum_{n} \frac{\partial A_{n}}{\partial \varphi} \cos n \lambda+\sum_{n} \frac{\partial B_{n}}{\partial \varphi} \sin n \lambda\right\} \\
v & =\frac{g \partial z}{f a \cos \varphi \partial \lambda} \\
& =-\frac{g}{f a \cos \varphi}\left\{\sum_{n} n A_{n} \sin n \lambda-\sum_{n} n B_{n} \cos n \lambda\right\} \\
\zeta & =\sum_{n} P_{n} \cos n \lambda+\sum_{n} Q_{n} \sin n \lambda
\end{aligned}
$$

where

$$
\begin{aligned}
P_{n} & =\nabla^{2} A_{n}, \quad Q_{n}=V^{2} B_{n} \\
\nabla^{2} & =\frac{g}{f a^{2} \cos \varphi}\left[\frac{\partial}{\partial \varphi}\left(\cos \varphi \frac{\partial}{\partial \varphi}\right)-\frac{n^{2}}{\cos \varphi}-\frac{a \beta}{f} \cos \varphi \frac{\partial}{\partial \varphi}\right] \\
\beta & =\frac{\partial f f}{a \partial \varphi}, \quad f=2 \omega \sin \varphi
\end{aligned}
$$


which expresses the vorticity change expected in the barotropic atmosphere, the time change of each coefficient of Fourier series, or that of spectrum of pressure field, is derived.

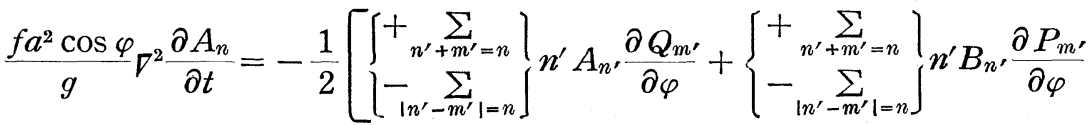

$$
\begin{aligned}
& \left.+\left\{\begin{array}{c}
-\sum_{n^{\prime}+m^{\prime}=n} \\
-\sum_{n^{\prime}-m^{\prime} \mid=n}
\end{array}\right\} \frac{\partial A_{n^{\prime}}}{\partial \varphi} m^{\prime} Q_{m^{\prime}}+\left\{\begin{array}{l}
-\sum_{n^{\prime}+m^{\prime}=n} \\
+\sum_{n^{\prime}-m^{\prime} \mid=n}
\end{array}\right\} \frac{\partial B_{n^{\prime}}}{\partial \varphi} m^{\prime} P_{m^{\prime}}\right]-\frac{\partial f}{\partial \varphi} \cdot n B_{n} \\
& \frac{f a^{2} \cos \varphi}{g} \nabla^{2} \frac{\partial B_{n}}{\partial t}=-\frac{1}{2}\left[\left\{\begin{array}{l}
-\sum_{n^{\prime}+m^{\prime}=n} \\
-\sum_{n^{\prime}-m^{\prime}=n} \\
+\sum_{n^{\prime}-m^{\prime}=-n}
\end{array}\right] n^{\prime} A_{n^{\prime}} \frac{\partial P_{m^{\prime}}}{\partial \varphi}+\left\{\begin{array}{c}
+\sum_{n^{\prime}+m^{\prime}=n} \\
-\sum_{n^{\prime}-m^{\prime}=n} \\
+\sum_{n^{\prime}-m^{\prime}=-n}
\end{array}\right\} n^{\prime} B_{n^{\prime}} \frac{\partial Q_{m}}{\partial \varphi}\right. \\
& \left.+\left\{\begin{array}{l}
+\sum_{n^{\prime}+m^{\prime}=n} \\
-\sum_{n^{\prime}-m^{\prime}=n} \\
+\sum_{n^{\prime}-m^{\prime}=-n}
\end{array}\right\} \frac{\partial A_{n^{\prime}}}{\partial \varphi} m^{\prime} P_{m^{\prime}}+\left\{\begin{array}{c}
-\sum_{n^{\prime}+m^{\prime}=n} \\
-\sum_{n^{\prime}-m^{\prime}=n} \\
+\sum_{n^{\prime}-m^{\prime}=-n}
\end{array}\right\} \frac{\partial B_{n^{\prime}}}{\partial \varphi} m^{\prime} Q_{m^{\prime}}\right]+\frac{\partial f}{\partial \varphi} \cdot n A_{n}
\end{aligned}
$$

Next, let us indicate the finite difference expressions of equation (2.3) in the following.

$$
\begin{aligned}
& \alpha_{+}(\varphi) \dot{A}_{n}(\varphi-\Delta \varphi)+\alpha_{-}(\varphi) \dot{A}_{n}(\varphi+\Delta \varphi)-\alpha_{0, n}(\varphi) \dot{A}_{n}(\varphi) \\
& =\frac{-10^{-3}}{2}\left[\left\{\begin{array}{c}
+\sum_{n^{\prime}+m^{\prime}=n} \\
-\sum_{\left|n^{\prime}-m^{\prime}\right|=n}
\end{array}\right\} n^{\prime} A_{n}, \boldsymbol{\delta}_{\varphi} q_{m^{\prime}}+\left\{\begin{array}{l}
+\sum_{n^{\prime}+m^{\prime}=n} \\
+\sum_{\left|n^{\prime}-m^{\prime}\right|=n}
\end{array}\right\} n^{\prime} B_{n^{\prime}} \boldsymbol{\delta}_{\varphi} q_{m},+\left\{\begin{array}{c}
-\sum_{n^{\prime}+m^{\prime}=n} \\
-\sum_{\left|n^{\prime}-m^{\prime}\right|=n}
\end{array}\right\} \boldsymbol{\delta}_{\varphi} A_{n^{\prime}} \cdot m^{\prime} \boldsymbol{q}_{m^{\prime}}\right. \\
& \left.+\left\{\begin{array}{l}
-\sum_{n^{\prime}+m^{\prime}=n} \\
+\sum_{\left|n^{\prime}-m^{\prime}\right|=n}
\end{array}\right\} \delta_{\varphi} B_{n} \cdot m^{\prime} p_{m},\right]-\gamma_{n} \cdot B_{n} \\
& p_{m^{\prime}}=\frac{10^{3} \Delta \varphi}{2 \cos \varphi} P_{m^{\prime}}, \quad q^{\prime} m=\frac{10^{3} \Delta \varphi}{2 \cos \varphi} Q_{m^{\prime}}
\end{aligned}
$$

where

with notations, which differ slightly from the notations in the foregoing paper;

$$
\begin{aligned}
& l=10^{-3} \frac{2 \omega a^{2}}{g}=22.8 \text { (feet-day) } \\
& \alpha_{+}(\varphi)=1+\Delta \varphi \operatorname{cosec} 2 \varphi, \quad \alpha_{-}(\varphi)=1-\Delta \varphi \operatorname{cosec} 2 \varphi, \quad \alpha_{0, n}(\varphi)=2+n^{2}(\Delta \varphi)^{2} \sec ^{2} \varphi \\
& \beta_{+}(\varphi)=\frac{\alpha_{+}}{l \Delta \varphi} \operatorname{cosec} 2 \varphi, \quad \beta_{-}(\varphi)=\frac{\alpha_{-}}{l \Delta \varphi} \operatorname{cosec} 2 \varphi, \quad \beta_{0, n}(\varphi)=\frac{\alpha_{0, n}}{l \Delta \varphi} \operatorname{cosec} 2 \varphi \\
& \gamma_{n}=2 \omega n(\Delta \varphi)^{2} \quad \text { and } \quad 2 \Delta \varphi=0.1748 \text { in our case. } \\
& {\left[\begin{array}{l}
\delta A_{n}(\varphi) \\
\delta B_{n}(\varphi)
\end{array}\right]=\left[\begin{array}{l}
A_{n}(\varphi+\Delta \varphi)-A_{n}(\varphi-\Delta \varphi) \\
B_{n}(\varphi+\Delta \varphi)-B_{n}(\varphi-\Delta \varphi)
\end{array}\right] } \\
& {\left[\begin{array}{l}
p_{m},(\varphi) \\
q_{m}(\varphi)
\end{array}\right]=\left[\begin{array}{l}
A_{m},(\varphi-\Delta \varphi) \\
B_{m},(\varphi-\Delta \varphi)
\end{array}\right] \beta_{+}(\varphi)+\left[\begin{array}{l}
A_{m},(\varphi+\Delta \varphi) \\
B_{m},(\varphi+\Delta \varphi)
\end{array}\right] \beta_{-}(\varphi)-\left[\begin{array}{l}
A_{m},(\varphi) \\
B_{m},(\varphi)
\end{array}\right] \beta_{0, m},(\varphi) } \\
& {\left[\begin{array}{l}
\delta p_{m},(\varphi) \\
\delta q_{m},(\varphi)
\end{array}\right]=\left[\begin{array}{l}
p_{m},(\varphi+\Delta \varphi) \\
q_{m},(\varphi+\Delta \varphi)
\end{array}\right]-\left[\begin{array}{l}
p_{m},(\varphi-\Delta \varphi) \\
q_{m},(\varphi-\Delta \varphi)
\end{array}\right]-2 \Delta \varphi \tan \varphi\left[\begin{array}{l}
p_{m},(\varphi) \\
q_{m},(\varphi)
\end{array}\right] }
\end{aligned}
$$

The expression for $\dot{B_{n}}$ is simillar.

The right hand side of the equation (2.4) can be computed with the initial data of $z$, and thus $\dot{A_{n}}$ and $\dot{B_{n}}$ are to be obtained by one dimensional relaxation.

\section{§ 3. Computational Instability}

Adopting the linearized vorticity equation

$$
\nabla^{2} z_{t}+\left(U-\frac{g}{f a} \frac{\partial z}{\partial \varphi}\right) \frac{\partial \nabla^{2} z}{a \cos \varphi \partial \lambda}+
$$




$$
\begin{array}{r}
+\frac{\partial z}{a \cos \varphi \partial \lambda} \frac{\partial}{a \partial \varphi}\left(\frac{g}{f} \nabla^{2} z-\frac{\partial U}{a \partial \varphi}\right) \\
=\beta \frac{\partial z}{a \cos \varphi \partial \lambda}
\end{array}
$$

with

$$
z \propto e^{i(n \lambda-\nu t)},
$$

we get the criterion of computational instability

$$
\Delta t \lesssim \frac{a \cos \varphi}{n}\left(U+\frac{a \cos ^{2} \varphi}{n^{2}}\left(\beta-\frac{\partial^{2} U}{a^{2} \partial \varphi^{2}}\right)\right)^{-1}
$$

It is noticed that the stability criterion (3.2), does not contain space decrement $a \Delta \varphi$ but wave number $n$, and thus it is sufficed if we calculate the maximum time interval allowed for the wave of the largest wave number ( $\boldsymbol{n}=6$, in our case). Generally speaking, the influence of $\partial^{2} U / a^{2} \partial \varphi^{2}$ is supposed to be small. Namely if $\partial^{2} U / a^{2} \partial \varphi^{2}$ can be neglected the equation (3.2) may be transformed into

$$
\Delta t \lesssim 1 / n\left(\omega_{0}+\frac{a \cos \varphi}{n^{2}} \beta\right)
$$

where

$$
\omega_{0} a \cos \varphi=U
$$

It is plausible from the equation (3.3) to assume $\Delta t=8 \mathrm{hrs}$ for $n=6, U=18 \mathrm{~m} / \mathrm{sec}, \beta=2.00$ $\times 10^{-13} \mathrm{~cm}^{-1} \mathrm{sec}^{-1}$.

Now we are going to carry out computation by hand owing to lack of high speed computing machine. Accordingly some method have to be deviced to make the time interval $\Delta t$ larger. For instance, the reduction of $U$ (by half) is useful for our purpose. Concerning this point, the concept of using moving coordinate was deviced by the staff members in Tokyo University. Making use of this method the time interval in one step is found to be allowed to be $16 \mathrm{hrs}$ or less, and in the practical calculation $\Delta t=8 \mathrm{hrs}$ is used for caution's sake.

\section{§. On the Vorticity Equation on the Mov- ing Coordinates with Constant Angular Velocity}

After the Staff Members in Tokyo University, the explanation of the vorticity equation on the moving coordinates with constant eastward angular velocity, will be shown in the following. Namely, the pressure field corresponding to the general current stated above only translates linearly the remaining pres- sure pattern, and has no non-linear coupling with them. Accordingly, if we put

$$
\begin{aligned}
& \lambda=\Lambda+\omega^{\prime} t, \\
& u=u^{\prime}+a \omega^{\prime} \cos \varphi, v=v^{\prime}
\end{aligned}
$$

the equation

$$
\begin{gathered}
\left(\frac{\partial \zeta^{\prime}}{\partial t}\right)_{\Lambda}+\frac{2 v^{\prime}}{a}\left(\omega+\omega^{\prime}\right) \cos \varphi+\frac{v^{\prime}}{a} \frac{\partial \zeta^{\prime}}{\partial \varphi} \\
+\frac{u^{\prime}}{a \cos \varphi} \frac{\partial \zeta^{\prime}}{\partial \Lambda}=0
\end{gathered}
$$

can be derived, where $\Lambda$ denotes the longitude of the air particle referred to the moving coordinates with constant eastward angular velocity $\omega^{\prime} . \quad u^{\prime}, v^{\prime}$ and $\zeta^{\prime}\left(\zeta=\zeta^{\prime}+2 \omega^{\prime} \sin \varphi\right)$ are the east and north-ward components of velocity, the vertical component of relative vorticity relative to the moving coordinates, respectively.

If we put

$$
z=z^{\prime}+z^{*}(\varphi), \quad \zeta^{*}(\varphi)=2 \omega^{\prime} \sin \varphi
$$

the relation

$$
z^{*}(\varphi)-z^{*}\left(\varphi_{1}\right)=\frac{\omega a^{2} \omega^{\prime}}{2 g}\left(\cos 2 \varphi-\cos 2 \varphi_{1}\right)^{\Delta)}
$$

is derived.

It can thus be said that to obtain the vorticity distribution after a time interval $\Delta t$ by use of the barotropic model as in our case, it is enough to translate the pattern with $\omega^{\prime} \Delta t$ and to superpose on the final pattern thus obtained the amount of vorticity change calculated from the equation (4.2).

They say that this method has the following advantages; the larger time interval of one step is allowed in calculation, because the maximum velocity of air particle is reduced by using the moving coordinates. In addition, when we adopt the method using Fourier series for a limitted region, the amount of leaving vorticity from the east boundary is inevitably the same with that of entering vorticity through the west boundary. Thus this may be the cause of errors in calculation in reference to the fixed coordinates in the case where there is cyclonic or anticyclonic vorticity on both sides, even when there is no error due to the error near boundary. Even in such a case the method using the moving

4) Notations $z^{*}$ and $\zeta^{*}$ shall not be confused with those in the introduction. 
coordinate is found to be useful, because, as well known in the field, the velocity of vorticity flow is said to be nearly $10^{\circ}$ longitude per day on an average, and we can in the moving area get rid of the above mentioned errors considerably.

\section{$\S 5$. Procedure of Calculation}

In this section, now, let us explain the details of practical procedure of calculation suc- cessively. We adopted, as the forecasting area, a moving domain enclosed at first by $75^{\circ} \mathrm{N}$ and $25^{\circ} \mathrm{N}$ latitude circles, $10^{\circ} \mathrm{W}$ and $170^{\circ} \mathrm{E}$ meridians as shown in Fig. 1, the longitudinal boundaries of which are moving eastward with the velocity of $10^{\circ}$ or $15^{\circ}$ longitude per day.

Now, the length of latitude circle becomes shorter with increasing latitude $\varphi$ until zero at $90^{\circ} \mathrm{N}$. Accordingly, the scale of vorticity

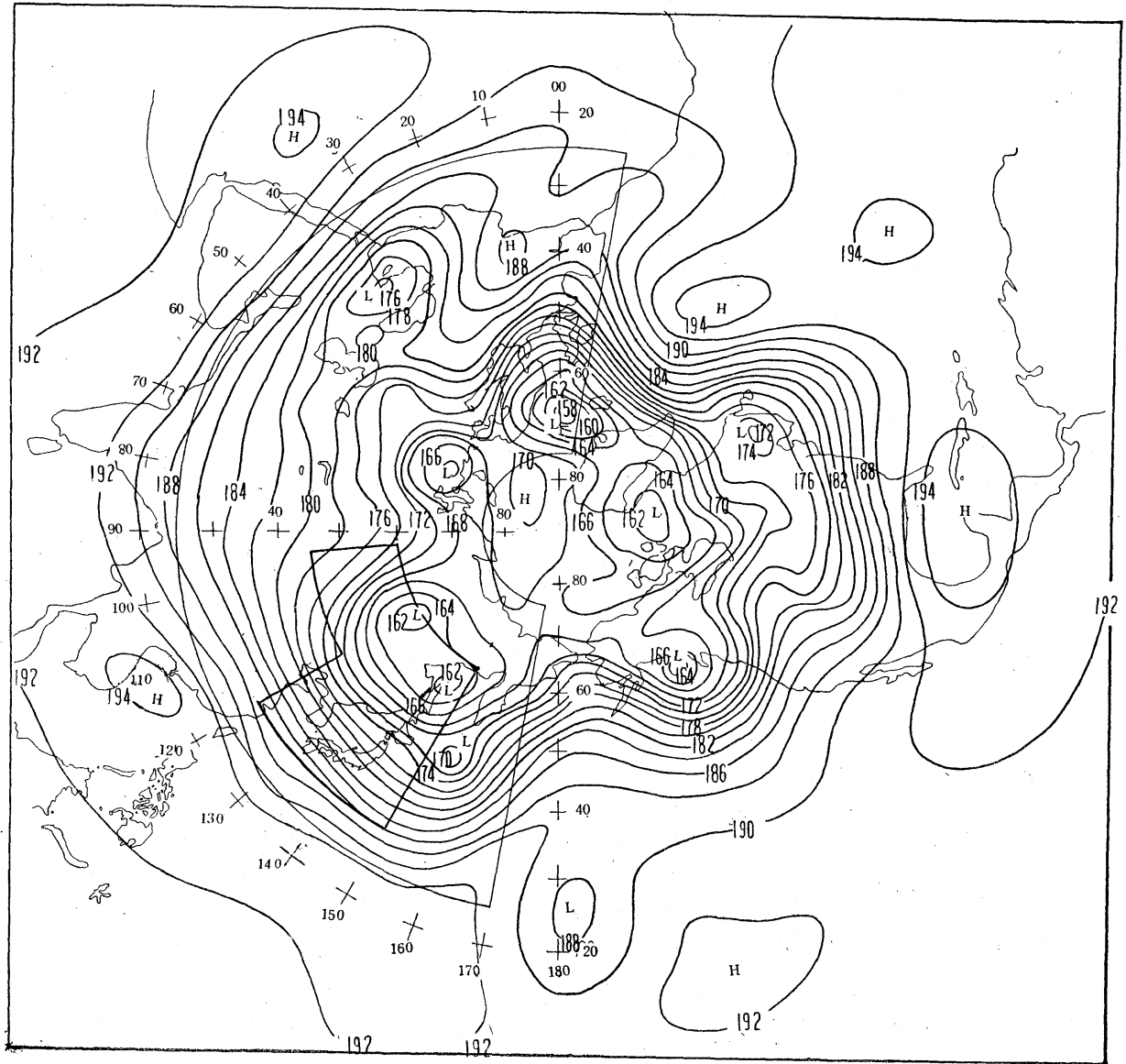

Fig. 1. Initial $500 \mathrm{mb}$ map, with height contours at $200 \mathrm{ft}$. intervals for $1500 \mathrm{GMT}$ on 9 , Jan. 1952. (in hundreds of feet). Thin solid lines on this chart enclose the initial location of the area moving eastward, and the heavy solid frame lines enclose the area of verification.

expressed by a certain wave, the wave number of which is fixed, seems to be too small compared with the scale of disturbances dealt with by us in high latitudes. To investigate this we tried to compare two kinds of vorticities, that is, those expressed by ours and those by conventional method. We denote the former $\zeta_{1}$ and the latter $\zeta_{2}$.
Then, if we put $z=A_{n} \cos n \lambda$,

$$
\begin{aligned}
\frac{f}{g} \zeta_{1}= & \frac{f}{g} P_{n} \cos n \lambda=\frac{1}{a^{2}(\Delta \varphi)^{2}}\left(\alpha_{+}(\varphi) A_{n}(\varphi-\Delta \varphi)\right. \\
& \left.+\alpha(\varphi) A_{n}(\varphi+\Delta \varphi)-\alpha_{0, n}(\varphi) A_{n}(\varphi)\right) \cos n \lambda \\
\frac{f}{g} \zeta_{2}= & \nabla^{2} z=\bar{z}-z=\frac{1}{(\Delta s)^{2}}\left\{\left(A_{n}(\varphi+\Delta \varphi)+\right.\right. \\
& \left.+A_{n}(\varphi-\Delta \varphi)-2 A_{n}(\varphi)\right) \cos n \lambda+
\end{aligned}
$$




$$
\begin{aligned}
& +A_{n}(\varphi)(\cos n(\lambda+\Delta \lambda) \\
& +\cos n(\lambda-\Delta \lambda)-2 \cos n \lambda)\} \\
D= & \frac{f}{g}\left(\zeta_{2}-\zeta_{1}\right) \quad \text { for } s=a \Delta \varphi \\
= & A_{n}(\varphi)\left\{2(\cos n \Delta \lambda-1)+\left(\frac{n \Delta \varphi}{\cos \varphi}\right)^{2}\right. \\
& \left.+\frac{\Delta \varphi}{\sin 2 \varphi} \frac{A_{n}(\varphi+\Delta \varphi)-A_{n}(\varphi-\Delta \varphi)}{A_{n}(\varphi)}\right\} \frac{\cos n \lambda}{(\Delta s)^{2}}
\end{aligned}
$$

As we are considering the difference of two expressions of vorticity at high latitudes, and $A_{n}(\varphi+\Delta \varphi)-A_{n}(\varphi-\Delta \varphi) \approx 0$ near the pole and the equater, the third term of the equation (5.1) may be neglected in practice. Then we calculated the correcting coefficients for $\zeta_{1}$ by use of the remaining two terms of the equation (5.1).

These coefficients times $(n \Delta \varphi / \cos \varphi)^{2}$ in $\alpha_{0, n}$

\section{Flow Diagram of Calculation}

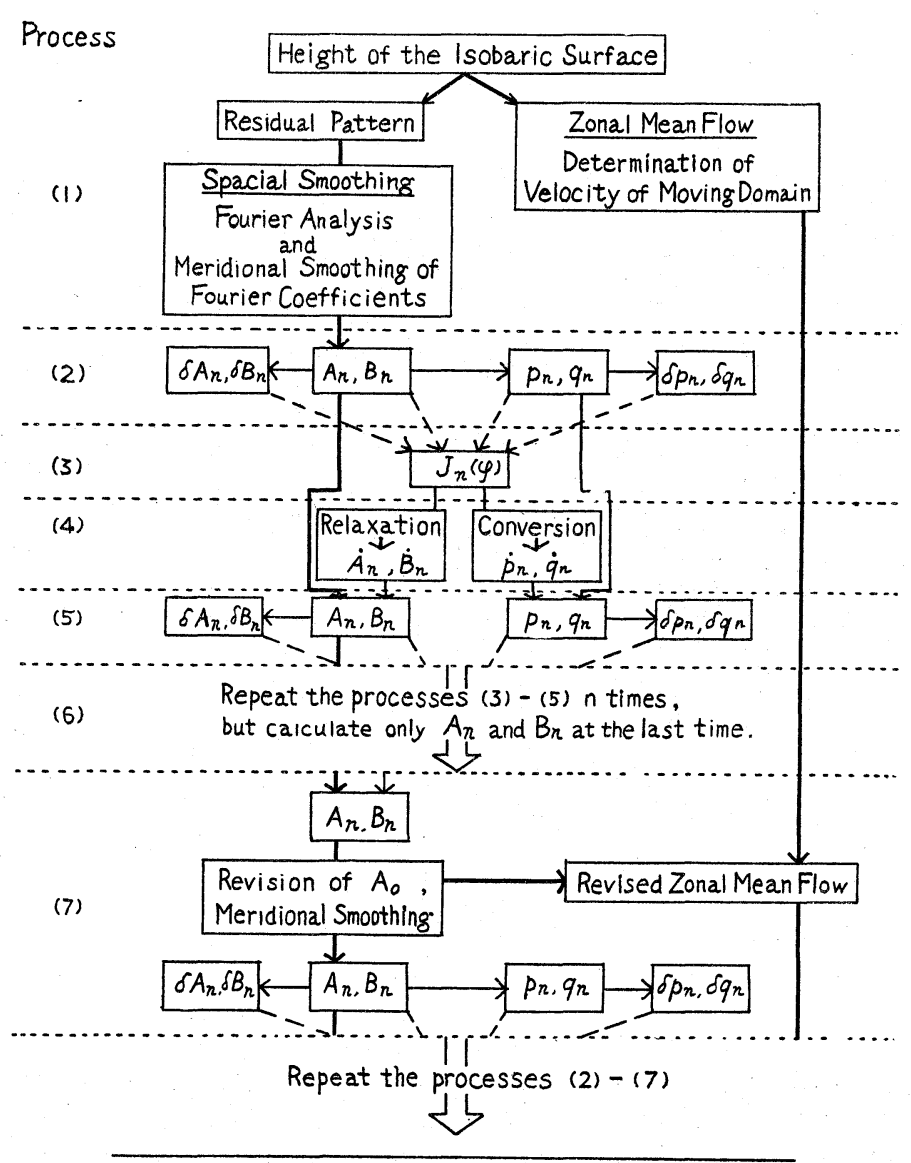

Remark: Revision of $A_{0}$ (process $(7)$ ) is omitted in our calculation.

or $\beta_{0, n}$ introduce the values of $p_{n} . q_{n}$ reasonably corresponding to the conventional method.

Then, we calculate the values of the right hand side of equation (2.4) for each wave, which corresponds to the so-called Jacobian, and we will show them by $J(\varphi)$ hereafter. We used the waves with wave numbers less than
3 in the mentioned domain.

To solve the equation (2.4), and obtain the changes of $A_{n}(\varphi)$ and $B_{n}(\varphi)$, the values of $J(\varphi)$ are relaxed by using the matrix for the wave number $n$ in question. These matrices are obtained as the reverse matrices of those corresponding to the left hand side of equa- 
Table 1. Correlation coefficients $(r)$ for barotropic $500 \mathrm{mb}$ forecasting at succesive period. $r$ 's in upper rank are those for figs. 2 (a), (b), (d) and (f), respectively, and $r$ 's in lower rank for figs. 2 (c), (e), (g) and for 15th, respectively.

\begin{tabular}{c|cccccc}
\hline $\begin{array}{c}\text { Computation } \\
\text { period }\end{array}$ & $\begin{array}{c}1 \\
(10 \mathrm{th})\end{array}$ & $\begin{array}{c}2 \\
(11 \mathrm{th})\end{array}$ & $\begin{array}{c}3 \\
(12 \mathrm{th})\end{array}$ & $\begin{array}{c}4 \\
(13 \mathrm{th})\end{array}$ & $\begin{array}{c}5 \\
(14 \mathrm{th})\end{array}$ & $\begin{array}{c}6 \\
(15 \text { th })\end{array}$ \\
\hline $\begin{array}{c}\text { Correlation } \\
\text { coefficient } r\end{array}$ & 0.72 & 0.80 & 0.67 & 0.02 & & days \\
\hline
\end{tabular}

Table 2. Zonal mean heighf of $500 \mathrm{mb}$ isobaric surface (in hundreds of feet, but the third digit is omitted) for every five latitudes in the moving area with eastward velocity $10^{\circ}$ longitudes per day, from 9 to 13, Jan. 1952.

\begin{tabular}{c|c|c|c|c|c|c|c|c|c|c|c|c|c|c|c}
\hline $\begin{array}{c}\text { Lati- } \\
\text { tude } \varphi\end{array}$ & 85 & 80 & 75 & 70 & 65 & 60 & 55 & 50 & 45 & 40 & 35 & 30 & 25 & 20 & 15 \\
\hline 9th & 69.6 & 69.3 & 68.1 & 65.2 & 66.3 & 68.3 & 71.2 & 74.4 & 77.4 & 80.0 & 82.5 & 85.9 & 89.1 & 91.4 & 93.4 \\
10th & & 68.1 & 66.5 & 65.2 & 66.3 & 69.3 & 71.7 & 73.3 & 76.2 & 79.2 & 82.3 & 85.5 & 88.9 & 91.5 & \\
11th & & 66.8 & 64.4 & 63.6 & 65.8 & 68.6 & 71.2 & 73.5 & 75.8 & 79.4 & 83.3 & 86.4 & 89.4 & 91.5 & \\
12th & & 66.3 & 65.1 & 65.5 & 67.4 & 70.2 & 73.0 & 74.8 & 77.3 & 80.4 & 83.8 & 86.8 & 89.6 & 91.8 & \\
13th & & 65.2 & 69.2 & 68.5 & 70.1 & 72.4 & 73.7 & 74.9 & 77.7 & 80.9 & 83.9 & 87.0 & 89.8 & & \\
\hline
\end{tabular}

tion (2.4), under the boundary condition; $\dot{A_{n}}$ $=\dot{B}_{n^{\prime}}=0$ at the north pole and the equator. For the waves with wave number $n>$ about 5 (in reference to $360^{\circ}$ longitudinal extension) the imposed boundary conditions have no influence on the values at middle latitudes of the matrices, but as for the large scale waves (wave number $n$ of which is smaller than 4) the boundary condition at the equator has an influence, which is not weak even at high latitudes, on the values of matrices.

To show the procedure of the calculation briefly, flow diagram is presented. With respect to procedure of computation, some remarks will be described.

$A_{n}(\varphi)$ 's and $B_{n}(\varphi)$ 's are smoothed meridonally at every time intervals, e.g., every 24 hours. This will be carried out by expanding $A_{n}(\varphi)$ 's into Fourier series along meridian from $0^{\circ} \mathrm{N}$ to $90^{\circ} \mathrm{N}\left(A_{n}(\varphi)\right.$ near the equator and the pole should be assumed to be zero, if unknown.) These smoothing means cutting off small scale disturbances in the direction of meridian produced by the couplings of waves. $\dot{A}_{n}(\varphi)$ 's and $\dot{\boldsymbol{B}}_{n}(\varphi)$ 's are obtained at every time interval.

$p_{n}(\varphi)$ 's and $q_{n}(\varphi)$ 's are not always computed directly, from $A_{n}(\varphi)$ 's and $B_{n}(\varphi)$ 's. The change of $p_{n}(\varphi)$ 's and $q_{n}(\varphi)$ 's are obtained from the value of $J(\varphi)$ 's. When $A_{n}(\varphi)$ 's and $B_{n}(\varphi)$ 's are smoothed meridionally, it is desirable to compute $p_{n}(\varphi)$ 's and $q_{n}(\varphi)$ 's from $A_{n}(\varphi)$ 's and

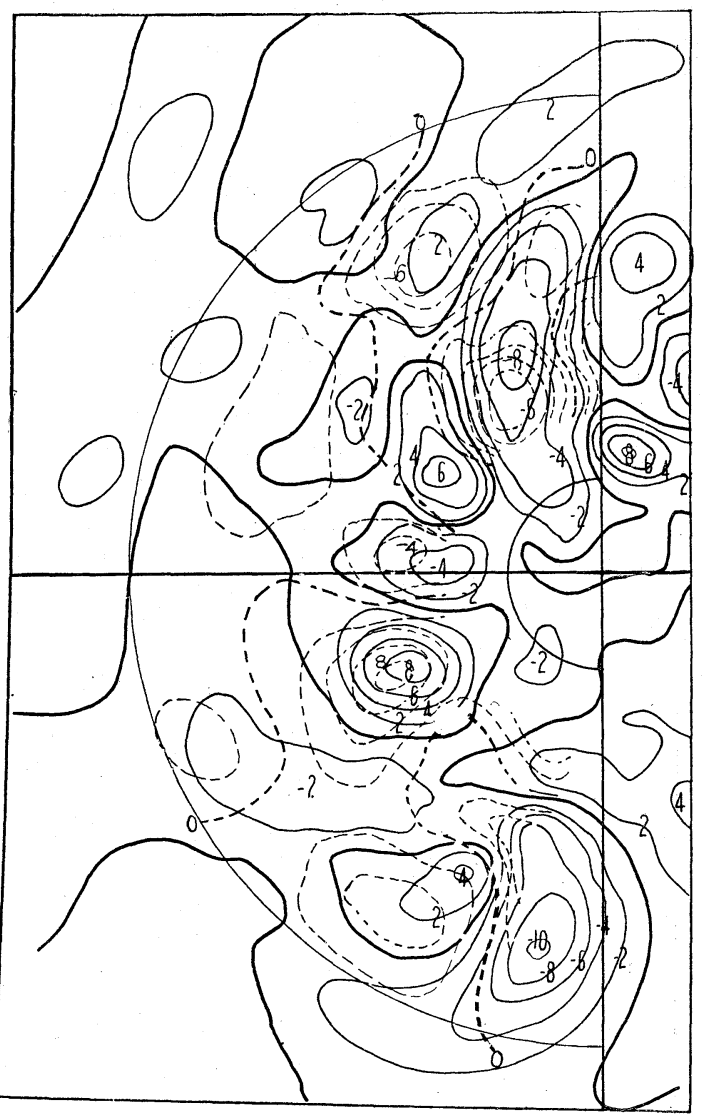

Fig. 2. (a) 
$B_{n}(\varphi)$ 's. The speed of zonal mean flow is generally $10^{\circ} \sim 15^{\circ}$ longitude or so eastward per day. We can revise the zonal mean flow, if we can predict the change of $A_{0}(\varphi)$ correctly.

\section{§ 6. An Example and Consideration on the Results}

Selecting $500 \mathrm{mb}$ chart among the Historical Weather Maps, as shown in fig. 1, we calculated height changes of $500 \mathrm{mb}$ isobaric surface on six successive days from January, 9th 1952. The solid lines in figures $2(a-h)$ show the observed values of change and the broken lines show the calculated ones, respectively.

In these figures, the moving sector enclosed by thin solid lines are forecasting area and the fixed smaller area (fig. 1) by heavy solid lines

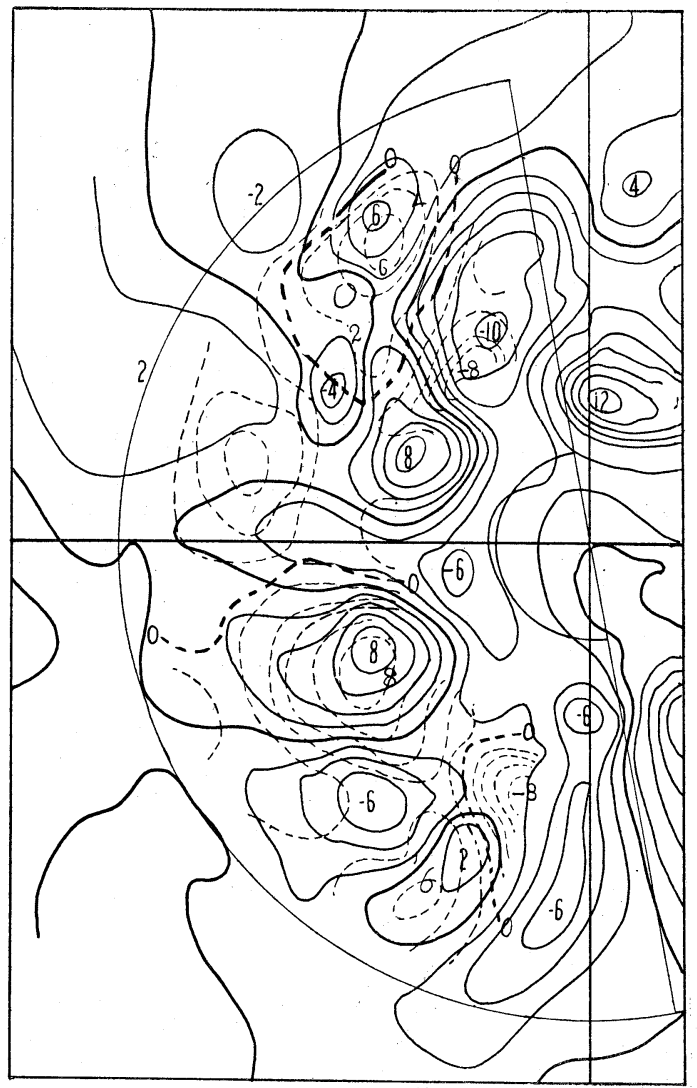

Fig. 2. (b) shows the area in which the values of correlation coefficients between the observed and computed height change of the isobaric surface are calculated. In these calculations the data at the 69 intersections of every five latitude and longitude circles are used.

Now, scrutinizing the figures $2(\mathrm{a}-\mathrm{f})$, the computation do not explain the weakening of the strong ridge located at the initial time far north of the Himalayas, over the Central Siberia, and west of the developed cyclones. Namely, the observed change amount to rising of eight hundred feet at the highest to east of the ridge, while calculated ones to one thousand five hundred feet on 12th, January. This tendency can already be recognized in the computation of the height change of 11th, and the relatively good results indicated by the correlation coefficient merely shows the

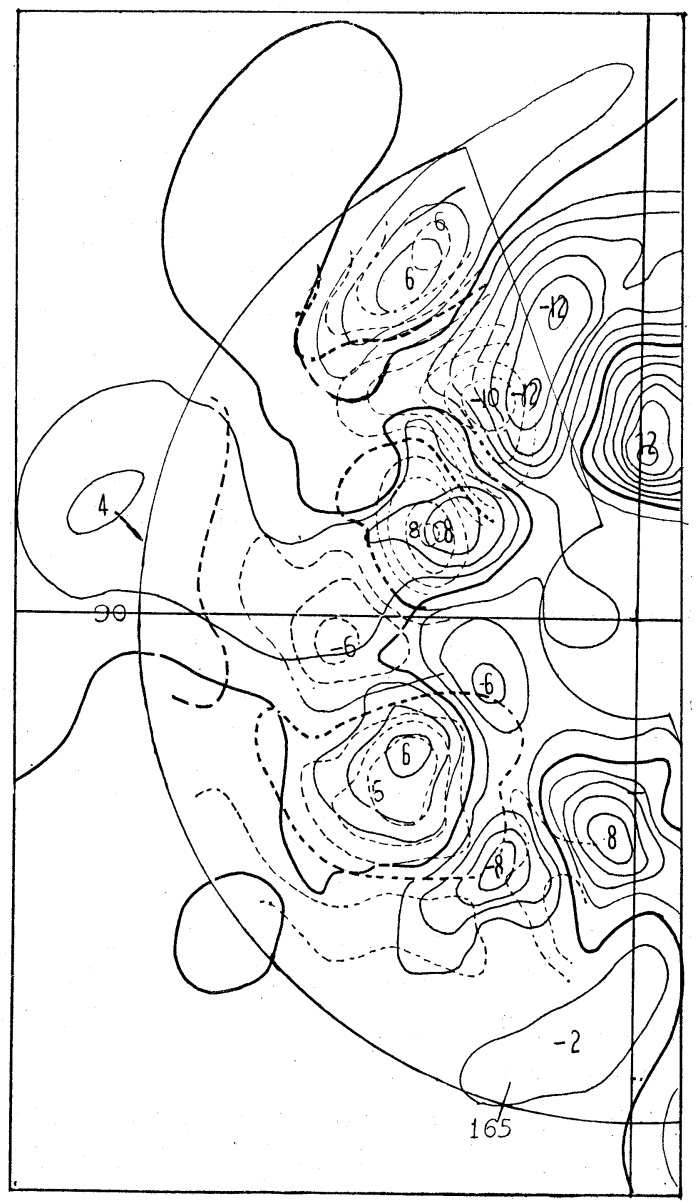

Fig. 2. (c) 


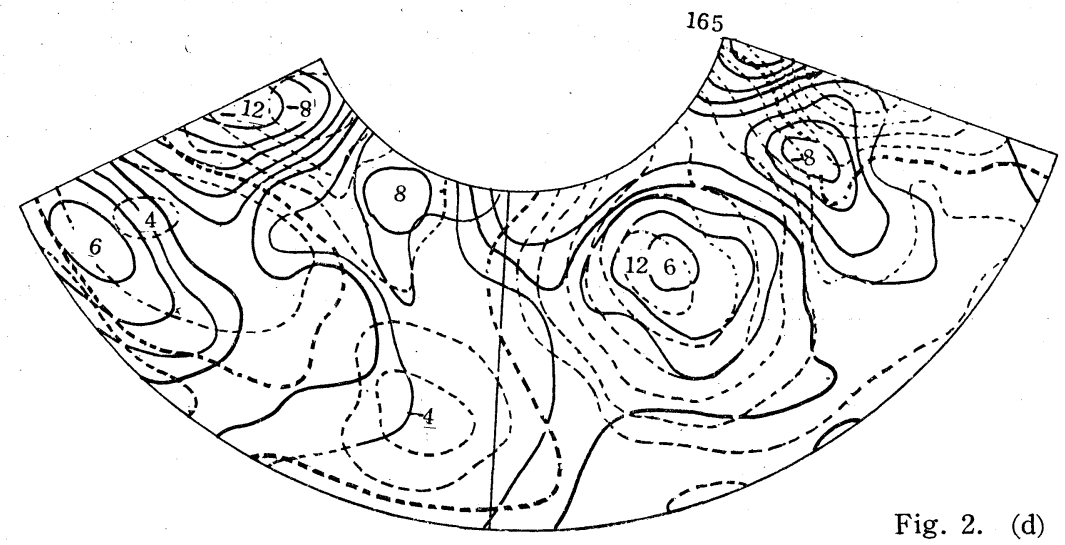

Fig. 2. (d)

90

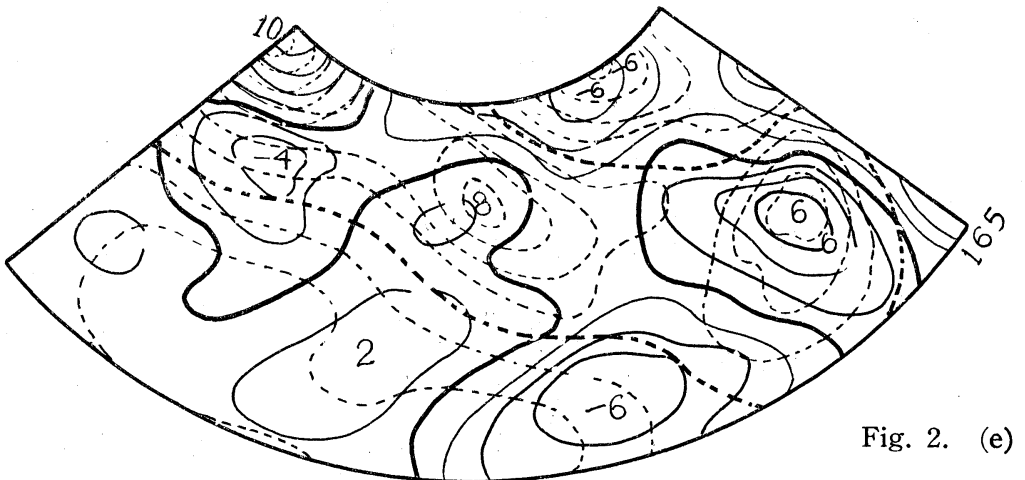

Fig. 2. Observed (continuous lines) and predicted (dashed lines) $500 \mathrm{mb}$ height changes in hundreds of feet for (a) I day period, (b) 2 day period, $(c$ and $d) 3$ day period, $(e$ and $f$ ) and 5 day period following the initial instant, respectively. For $(a)$, (b), $(d)$ and $(f)$, zonal mean flow at the initial instant was used throughout the calculation, while, for $(c),(e)$ and $(f)$, actual zonal mean flow for each day was used.

coincidence of pattern of height change and little about deepening. Then we investigated the change of $A_{0}(\varphi)$ or zonal mean flow, which is shown in table 2 . These values are obtained in the moving area with eastward velocity $10^{\circ}$ longitude/day.

It can easily be seen from this table that the amount of change in the mean zontal flow is little in the low latitudes and great in the high latitudes, especially on 12th and 13th.
The reason is that the predominant ridge extended towards north over the central Pacific, and this caused so-called blocking phenomena. In order to study these development, we plotted the locations and depths of each cyclone during this period in figure 3 ; the eastward velocity of the moving cyclones over the Siberia becomes less gradually and at last stagnant over the Okhotsk Sea and another small cyclone moves around along 
the periphery of that predominant ridge. Our calculation do not explain these movements. The variation of the mean zonal flow is not taken into account in our calculation at first, because our trials failed, as mentioned before, in predicting its variation owing to the bad convergency of local effect due to the method using harmonics, or improper model adopted by us, both of which are supposed to be blamed. Hereupon we tried to calculate the height change on 12th from the calculated data for 11th by use of the mean zonal flow obtained from observed values of $A_{0}(\varphi)$ in table 2. The revised value thus obtained (shown in figure 2 (c)) is found to be remarkably improved as compared with the centre and depths of each cyclone. The figures show the depth of cyclone (in hundreds of feet and the third digit is omitted), and those in parentheses indicate the date.
Fig. 3. The locations of

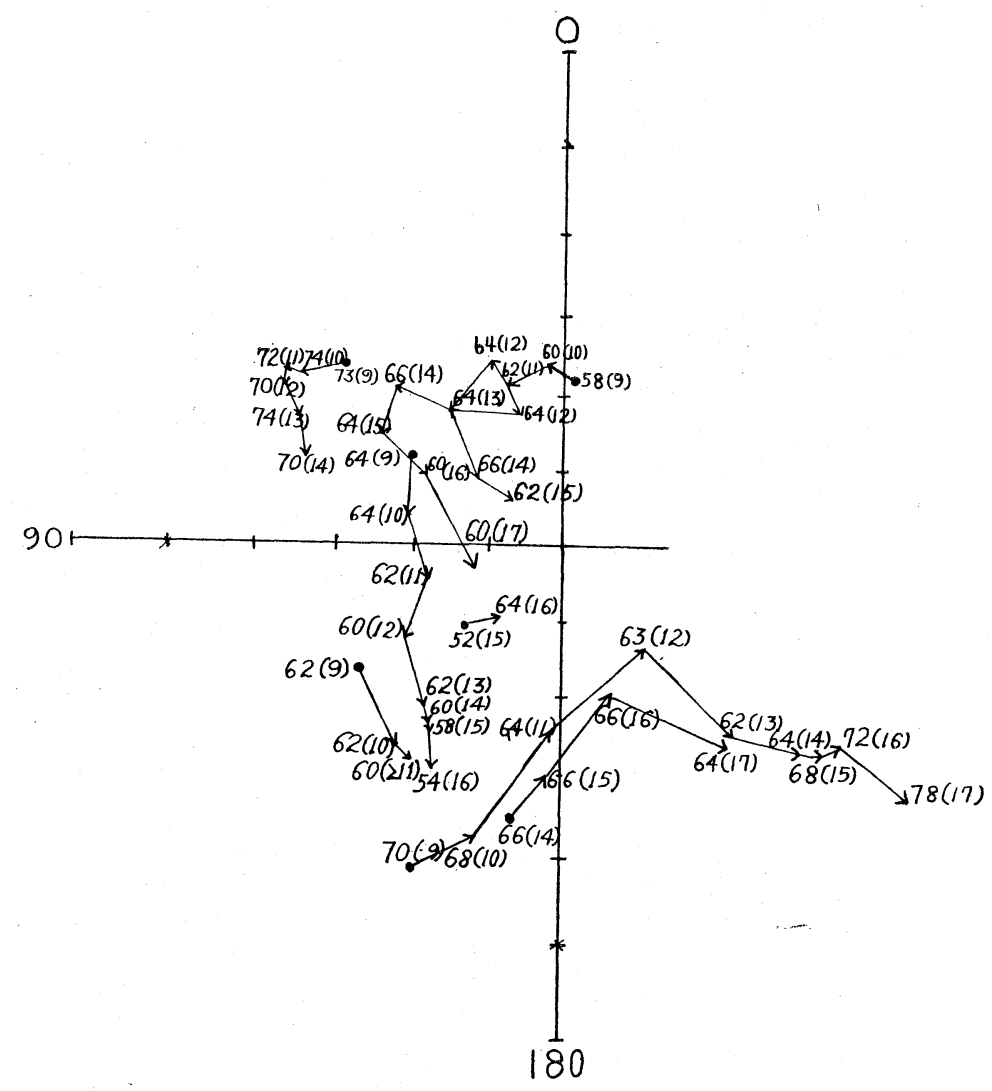

computation by use of initial $A_{0}(\varphi)$. The correlation coefficients $r$ in the upper rank of table 1 are obtained for the forecasting with computation by use of initial $A_{0}(\varphi)$ and the values $r$ in the lower rank are obtained for the one using observed $A_{0}(\varphi)$. The forecastings are found to be improved remarkably in latter cases, though the correction for change of vorticity in the field of $A_{0}(\varphi)$ is not taken into account here for the sake of simplicity. Accordingly it may be concluded that we can extend the forecasting period to about a week if the prediction of $A_{0}(\varphi)$ becomes possible and successful. This promises us the good future in the medium range forecasting through the numerical weather prediction, and recently a noteworthy study on the maintenance of $A_{0}(\varphi)$ was suggested by Akio Arakawa. He deals with the possibility of calculating the amount of the change in $A_{0}(\varphi)$ from the combination of two effects due to eddy horizontal transfer of momentum and eddy vertical transfer of potential temperature. Namely, the introduction of baroclinic effect of disturbances into our model through the change of $A_{0}(\varphi)$ will be made possible by using his method.

Further, as mentioned before, the predic- 
tion of the variation of the intensity of ridge over the central Siberia was failed. The height change for this ridge is rapid and it is difficult for us to forecast it with the barotropic model. It requires perhaps to introduce the effect of divergence, e., g., the effect due to topography.

\section{Acknowledgements}

The writers wish to express their thanks to the staff members of the Numerical Weather Prediction Group in Tokyo, for their many valuable advices and encouragements, in particular to Prof. S. Syōno, Dr. K. Gambo, Mr. K. Miyakoda and Mr. S. Manabe. And, moreover, we acknowledge here that the kind understanding and encouragements of Dr. K. Takahashi and Dr. R. Saito are indispensable for this study, and lastly that this work is obliged to the financial sponsorship of the Asahi.

\section{References}

1. Kubota, S., (1954); On the Numerical Prediction by the Quasi-double Fourier Series. Papers in Meteorology and Geophysics. 5, 144-152.

2. Gambo, K. and M. Aihara (1954); On Preliminary Investigation for the Long Range Fore- casting. (in Japanese). Technical Reviews. 5, 227-232.

3. Arakawa, Y. (1954); Examples of 36 hour Forecasting by use of Fj $\phi$ rtoft's Method. (in Japanese). Technical Reviews. 5, 178-179.

4. Kurihara, Y., (1955); Regional Numerical Weather Prediction in a Barotropic Atmosphere by the Method of One-dimensional Fourier Series with One-dimensional Relaxation. The Jour. Meteor. Soc. Japan, 33, 145-152.

5. Masuda, Y. (1955); On a Graphical Integration of the Barotropic Vorticity Equation and its Application to the Numerical Weather Forecast. (in Japanese). Jour. Meteor. Soc. Japan. 33, 280-290.

6. Sasaki, Y. (1955); A Fundamental Study of the Numerical Prediction Based on the Variational Principle. Jour. Meteor. Soc. Japan. 33, 262275.

7. Gambo, K. (1955); Preliminary Investigations for the Long Range Forecasting (unpublished).

8. Iida, M., N. Nakamura, and S. Kubota, (1956); On the Mean Spectral Distribution of the Height of $500-\mathrm{mb}$ Isobaric Surface along the $50^{\circ} \mathrm{N}$ Latitude Circle. Papers in Meteorology and Geophsics. 6, 291-295.

9. Uchiyama, H.; On the Study of Conservation of Absolute Vorticity (unpublished).

10. Staff Members in Tokyo University; Numerical Prediction using Moving Coordinate System. (to be published). 